\title{
Steady-state migrating intestinal dendritic cells induce potent inflammatory responses in naive CD4 ${ }^{+}$T cells
}

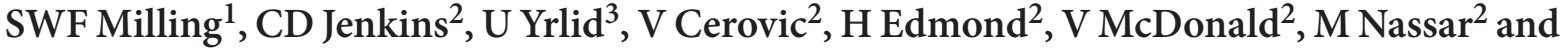 \\ G MacPherson ${ }^{2}$
}

Steady-state dendritic cells (DCs) migrating in the lymph from the intestine induce tolerance to harmless intestinal antigens, preventing inflammatory responses. To determine if such DCs are inherently tolerogenic we collected intestinal lymph DCs (L-DCs) by cannulation of the thoracic duct of rats after mesenteric lymphadenectomy, and examined their capacity to activate naive CD4 + lymphocytes in an allogeneic mixed leucocyte reaction. L-DCs stimulated strong proliferative responses, induced secretion of inflammatory cytokines including interferon- $\gamma$, and induced FoxP3-positive lymphocytes to divide. To determine if the activated $\mathrm{CD}^{+} \mathrm{T}$ cells had been tolerized, they were rested and restimulated with irradiated splenocytes. The restimulated $\mathrm{CD} 4^{+} \mathrm{T}$ cells again proliferated and secreted inflammatory cytokines. These data demonstrate that the DCs, which migrate from the intestine in the steady state, are paradoxically able to induce strong inflammatory responses from naive T cells, despite their role in the maintenance of oral tolerance.

\section{INTRODUCTION}

The intestinal immune system maintains a delicate and dangerous balancing act; the same tissues and cell populations are responsible for eliciting both strong protective immune responses following interaction with pathogens and for maintaining tolerance to harmless food and self-antigens. ${ }^{1}$ The dendritic cells (DCs) that migrate constitutively from the intestine to the mesenteric lymph nodes (MLNs) 2,3 have a "semimature" phenotype, expressing high levels of surface major histocompatibility complex (MHC) class II molecules, but only low levels of costimulatory molecules such as CD80 and CD86. ${ }^{4}$ In a model system, DCs of a similar phenotype generated from precursors in culture can induce allogeneic T lymphocytes to differentiate into populations expressing interleukin (IL)-13 and IL- 5 but not interferon (IFN)- $\gamma$, tumor necrosis factor- $\alpha$, or IL- $1 \beta .^{5}$ The soluble cytokine thymic stromal lymphopoietin (TSLP) can "condition" monocyte-derived DCs to elicit this T-cell phenotype. ${ }^{5,6}$ The inference from this study is that constitutively migrating DCs, conditioned by TSLP when resident in the intestinal lamina propria, are unable to stimulate inflammatory responses from naive $\mathrm{T}$ lymphocytes. This is an attractive hypothesis as it could explain why, under steady-state condi- tions, DCs can interact with naive T cells without inducing active immunity. However, interactions between intestinal DCs and naive $\mathrm{T}$ cells do not occur in the lamina propria but in the draining lymph nodes.

Steady-state migrating lymph DCs (L-DCs) are the cells that are able to initiate intestinal immune responses ${ }^{7}$ and are required for the maintenance of oral tolerance. ${ }^{8} \mathrm{~L}-\mathrm{DC}$ can be separated into at least three subsets with distinct functional properties. Two subsets were originally described, differentiated by their expression of CD4 or CD172a (SIRP $\alpha) .{ }^{9}$ The $\mathrm{CD} 4{ }^{\text {hi }} \mathrm{CD} 172 \mathrm{a}^{\text {hi }}$ subset stimulated stronger proliferation from enriched allogeneic $\mathrm{CD} 4^{+} \mathrm{T}$ cells and was better able to prime responses against $\mathrm{KLH}$ in vivo. ${ }^{9}$ The $\mathrm{CD} 4{ }^{\text {lo }} \mathrm{CD} 172 \mathrm{a}^{\text {lo }} \mathrm{L}-\mathrm{DC}$ subset appears to transport fragments of apoptotic enterocytes to the draining lymph nodes and has been implicated as being more important for the maintenance of oral tolerance. ${ }^{10}$ More recently, $\mathrm{CD} 11 \mathrm{~b}$ has been used as a marker to further separate the CD $4{ }^{\text {hi }}$ CD172a ${ }^{\text {hi }}$ L-DC subset into two populations. ${ }^{11}$ The $\mathrm{CD} 4{ }^{\text {hi }} \mathrm{CD} 172 \mathrm{a}^{\text {hi }} \mathrm{CD} 11 \mathrm{~b}$ hi ("CD $11 \mathrm{~b}$ hi") L-DCs and the CD 4 hi CD172ahi CD11b ("CD172ahi”) L-DCs are functionally different; CD11b $1 b^{\text {lo }}$ L-DCs secrete higher levels of IL12p40 and IL-6 after stimulation through a TLR ligand combined with

\footnotetext{
${ }^{1}$ Glasgow Biomedical Research Centre, University of Glasgow, University Place, Glasgow, UK; ${ }^{2}$ Sir William Dunn School of Pathology, Oxford University, Oxford, UK ${ }^{3}$ Department Microbiology and Immunology, University of Gothenburg, Gothenburg, Sweden. Correspondence: SWF Milling (s.milling@clinmed.gla.ac.uk) 
CD40-specific antibodies. ${ }^{11}$ To understand the roles of these migrating DC populations in maintaining tolerance to intestinal antigens, we tested whether they are conditioned to stimulate noninflammatory $\mathrm{Th} 2$ responses or induce tolerance, as had been proposed.

We collected L-DCs by thoracic duct cannulation of mesenteric lymphadenectomized rats. ${ }^{2}$ L-DCs were purified into subsets by magnetic bead enrichment and MoFlo sorting, and used to stimulate purified naive allogeneic $\mathrm{CD} 4^{+}$lymphocytes. Lymphocyte proliferation and cytokine production were analyzed to determine the nature of DC-induced T-cell responses. DC-stimulated lymphocytes were also rested and restimulated to explore whether they had become polarized or even nonresponsive after interaction with migrating intestinal DCs.

The results demonstrate that naive $\mathrm{T}$ cells are activated by all three L-DC subsets; they proliferate strongly and secrete inflammatory and Th1 cytokines. Populations of both $\mathrm{CD} 4{ }^{+} \mathrm{CD} 25^{+} \mathrm{FoxP}_{3}{ }^{-}$and $\mathrm{CD} 4{ }^{+} \mathrm{CD} 25^{+} \mathrm{FoxP}^{+}{ }^{+} \mathrm{T}$-cells expand after interaction with the L-DCs. The stimulated T cells do not become tolerant or nonresponsive; when rested and restimulated they proliferate and again produce inflammatory and Th1 cytokines. Overall, these results challenge our current understanding of the role of these cells by showing that L-DCs are not conditioned to induce a noninflammatory $\mathrm{Th} 2$ phenotype in naive $\mathrm{T}$ cells. Finally, we suggest mechanisms by which tolerance to harmless intestinal antigens may be maintained despite the immunostimulatory phenotype of L-DCs.

\section{RESULTS \\ CD172a ${ }^{\text {hi }}$ migrating intestinal lymph DCs stimulate strong proliferation from allogeneic $\mathrm{CD} 4{ }^{+} \mathrm{T}$ cells}

To test whether the DCs that migrate from the intestine have the same properties as those expanded from precursors in vitro, we collected DCs from pseudo-afferent intestinal lymph by cannulation of the thoracic duct of rats after mesenteric lymphadenectomy. ${ }^{2}$ Intestinal L-DCs from PVG strain rats (RT1 ${ }^{\mathrm{c}}$ haplotype) were enriched using magnetic beads and purified by MoFlo sorting, based on high surface expression of CD103 and MHC class II molecules, and sorted into the three functionally distinct subsets by their expression of CD172a and CD11b. ${ }^{11}$ Representative dot plots illustrating the gating strategy used to purify L-DCs (Figure 1a) and the three L-DC subsets (Figure 1b) are shown. All the DCs in rat thoracic duct lymph express CD103 and high levels of surface MHC class II molecules (Figure 1a, b, and ${ }^{11}$ ). To measure their ability to activate $\mathrm{CD} 4^{+} \mathrm{T}$ cells in the steady state, L-DCs of each subset were initially cultured with sorted $\mathrm{CD}^{+} \mathrm{T}$ cells from the lymph nodes or spleens of DA (RT1 $\left.{ }^{\mathrm{a}}\right)$ or PVG-RT1 ${ }^{\mathrm{u}}$ animals, and the T-cell proliferation assessed by measuring tritiated thymidine incorporation 5 days later. Both CD172a hi L-DC subsets stimulated vigorous proliferative responses from MoFlo-purified CD4 ${ }^{+} \mathrm{T}$ cells (Figure 1c). This $\mathrm{CD} 4^{+}$population contains a mixture of naive, activated, memory and regulatory $\mathrm{T}$ cells. To assess the effects of the three subsets of L-DCs on the different $\mathrm{CD} 4^{+} \mathrm{T}$-cell populations, we first removed the $\mathrm{CD}_{25}{ }^{+}$(activated/regulatory) T cells from the $\mathrm{CD} 4^{+} \mathrm{T}$-cell pool by MoFlo sorting. This increased the prolif- erative responses from T-cells stimulated with CD172 $\mathrm{a}^{\text {hi }} \mathrm{DCs}$ (Figure 1d). Secondly, we separated $\mathrm{CD} 45 \mathrm{RC}^{\mathrm{lo}} \mathrm{CD} 25^{-} \mathrm{CD} 4^{+}$ $\mathrm{T}$ cells (memory phenotype) from CD45RC ${ }^{\text {hi }} \mathrm{CD} 25^{-} \mathrm{CD} 4{ }^{+} \mathrm{T}$ cells (naive phenotype). Allo-DCs stimulated more proliferation from the CD45RC ${ }^{\text {lo }}$ cells than from their CD45RChi naive counterparts (Figure 1e, f). In all cases, the CD172a ${ }^{\text {lo }} \mathrm{L}-\mathrm{DC}$ subset of L-DCs stimulated lower levels of proliferation than either of the CD172a $\mathrm{a}^{\text {hi }} \mathrm{L}-\mathrm{DC}$ subsets.

\section{L-DC-stimulated naive CD4 + T-cells generate inflammatory and Th1 cytokines}

To assess cytokine secretion by activated T cells, naive MoFlosorted $\mathrm{CD} 4{ }^{+}\left(\mathrm{CD} 25^{-} \mathrm{CD} 45 \mathrm{RC}^{\mathrm{hi}}\right)$ lymphocytes were mixed with purified steady-state DCs from each of the three subsets and supernatants sampled after 3, 4, and 5 days and analyzed for cytokine production. Samples from wells containing 2,000 DCs were compared. All three subsets of DCs induced the secretion of IL-2 and IFN- $\gamma$ (Figure 2a). On one occasion, CD172 $\mathrm{a}^{\text {lo }} \mathrm{L}-\mathrm{DC}$ also induced expression of IL-10 (Figure 2a). All the DC subsets also induced low levels of expression of IL-6, tumor necrosis factor- $\alpha$, and IL-9, but IL- 17 was not detected in any of the wells (data not shown). No detectable cytokines were secreted by $\mathrm{T}$ cells or DCs cultured alone (data not shown). When MoFlosorted naive T cells were stimulated by $1 \times 10^{5}$ allogeneic splenocytes in the presence of $1 \times 10^{5}$ additional MoFlo-sorted B cells (Igא + or Ig $\lambda+$ lymphocytes), they consistently produced IL-10 ( $42.44 \mathrm{pg} / \mathrm{ml}$, standard deviation 10.40). To assess the proportion of T-cells producing IL-2 and Th1 cytokines we performed mixed leucocyte reactions (MLRs) in enzyme-linked immunospot assay (ELISPOT) wells and counted the numbers of IL-2and IFN- $\gamma$-producing cells. These experiments demonstrated that $0.5-1.5 \%$ of the input number of T-cells secrete IL-2 during the MLR, and $0.05-0.1 \%$ of the T-cells secrete IFN- $\gamma$ (Figure 2b), depending on the L-DC subset used as APCs. The ratio of IFN- $\gamma$-/IL-2-producing T-cells calculated from these results is approximately 1:20, and does not differ significantly between L-DC subsets (Figure 2c).

\section{Both $\mathrm{CD}^{2} 5^{+} \mathrm{FOXP}^{-}$and $\mathrm{CD}^{-} 5^{+} \mathrm{FOXP}^{+} \mathrm{T}$ cells are expanded in MLR cultures}

To further analyze T-cell proliferation, sorted allogeneic CD4 ${ }^{+}$ CD45RChi $\mathrm{CD}^{2} 5^{-} \mathrm{T}$ cells were labeled with carboxyfluorescein succinimidyl ester (CFSE) and cultured with purified DC subsets. CFSE dilution revealed proliferation among T-cells stimulated by all of the L-DC subsets, consistent with the thymidine incorporation results shown above. Flow cytometric analysis showed that most of the proliferating cells expressed the high-affinity IL-2 receptor, CD25 (Figure 3a). This proliferating CD25-expressing population comprised approximately $25 \%$ of all $\mathrm{CD}^{+}$cells by day 3 , and was generated from MoFlo-purified $\mathrm{CD} 4^{+} \mathrm{CD} 25^{-} \mathrm{T}$ cells, containing fewer than $2 \% \mathrm{CD}_{2} 5^{+}$cells, and fewer than $0.2 \% \mathrm{FoxP}^{+}$cells (data not shown).

A proportion (14 $\pm 7 \%$ ) of these CD25-positive proliferating T-cells expressed the FoxP3 transcription factor (Figure 3b), suggesting that they were of a regulatory phenotype. Counting 
a
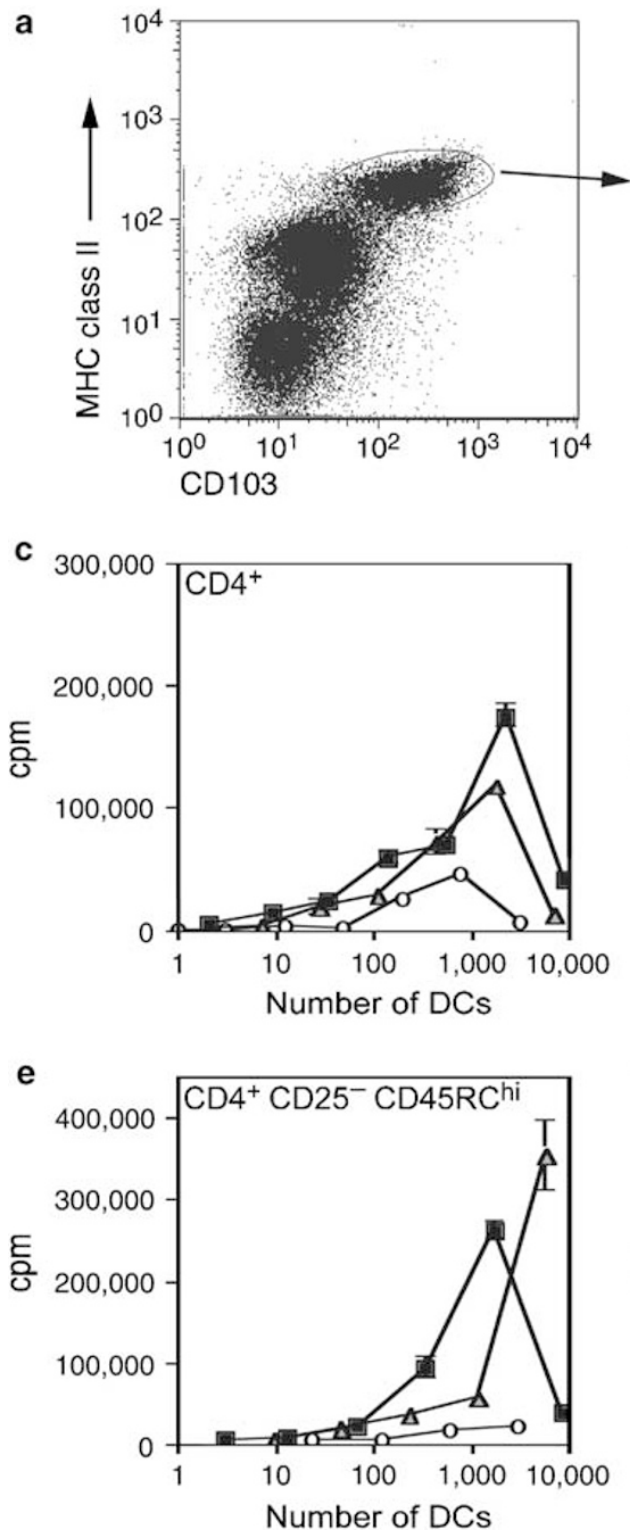

b

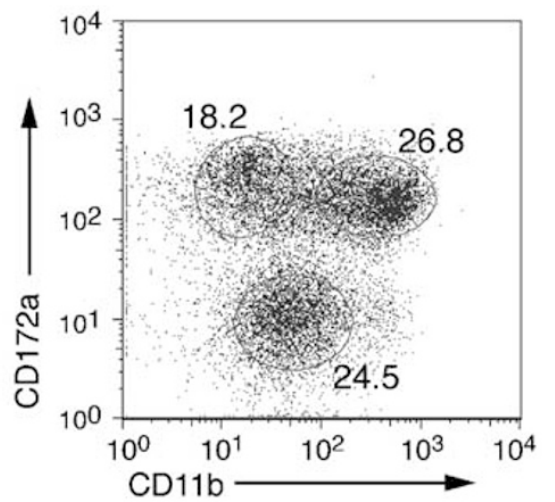

d

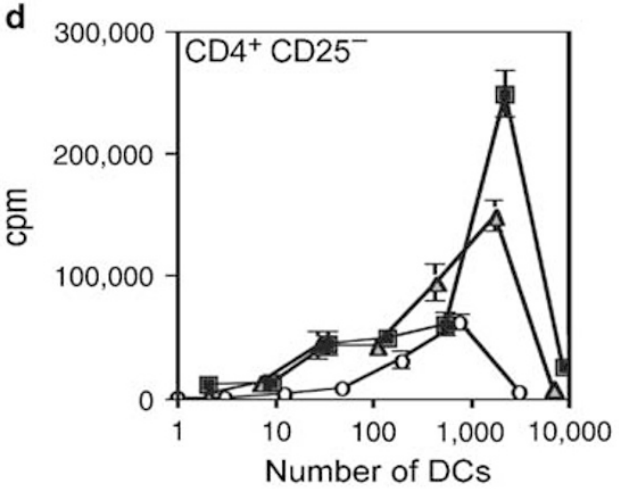

f

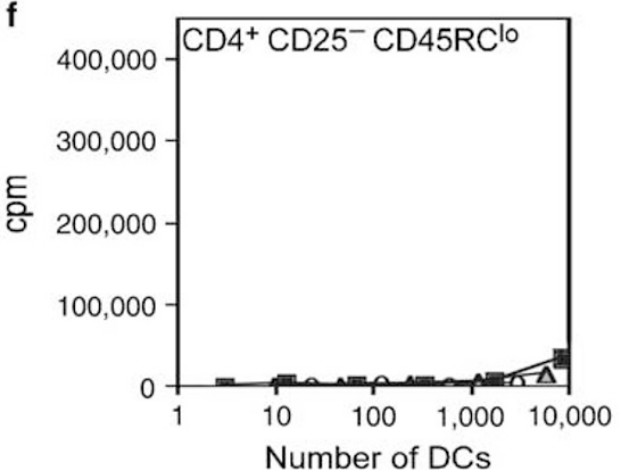

Figure 1 Steady-state DCs migrating from the intestine stimulate strong proliferative responses from naive allo-T cells. (a) Intestinal lymph DCs were identified by their expression of CD103 and high levels of MHC class II. (b) Subsets were identified by their differential expression of CD172a and CD11b. Numbers represent the percentage of CD103+ MHC class II hi sorted cells in each subset. MoFlo-sorted L-DC subsets from PVG-RT1 ${ }^{\mathrm{C}}$ rats were titrated and mixed with $1 \times 10^{5}$ MoFlo-sorted lymphocytes from DA rats. Lymphocytes were sorted according to the following criteria: (c) CD4 ${ }^{\text {hi }}$

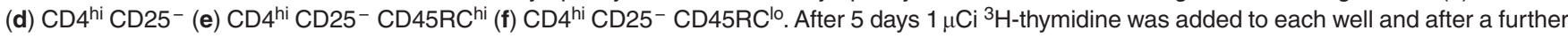
$18 \mathrm{~h}$ the amount of isotope incorporated into cells' DNA was measured by scintillation counting. T cells were stimulated with the following subsets of L-DCs: CD172a hi (black-filled squares), CD11b ${ }^{\text {hi }}$ (gray-filled triangles), CD172alo (open circles). Removal of the CD25+ ${ }^{+}$-cells significantly increased proliferative responses from T-cells stimulated with CD172ahi DCs $(P=0.01$, one-tailed paired $t$-test; $\mathrm{d})$. Error bars represent the standard error of the mean calculated from triplicate wells in one experiment. Data are representative of at least three experiments. DC, dendritic cell; MHC, major histocompatibility complex.

of cells recovered from the MLR reactions revealed that as expected, L-DC subsets stimulated an increase in the number of "activated" CD25 ${ }^{+}$FoxP3 ${ }^{-}$cells during the cultures. The CD172 $\mathrm{a}^{\text {hi }}$ and CD11b hi L-DC subsets stimulated the highest levels of proliferation (Figure 3c). All three DC subsets also stimulated an increase in the number of "regulatory" CD25 FoxP3 ${ }^{+}$cells in the cultures, although the CD11 b ${ }^{\text {hi }}$ L-DCs again induced the highest numbers (Figure 3d).

\section{When restimulated, L-DC-stimulated naive CD4 + T-cells generate inflammatory, and Th1 cytokines}

To test whether the allo-specific $\mathrm{T}$ cells became polarized after the initial stimulation, we cultured naive $\mathrm{CD} 4^{+} \mathrm{T}$ cells with $\mathrm{DCs}$ at a $\mathrm{T}$ cell/DC ratio 10:1. After 5 days $\mathrm{T}$ cells were rested in a low concentration of IL-2 $(20 \mathrm{U} / \mathrm{ml})$ for 3 days before restimulating the surviving cells in one of three ways. Firstly, we used irradiated $\mathrm{RT} 1^{\mathrm{c}}$ splenocytes from PVG rats, syngeneic with the 

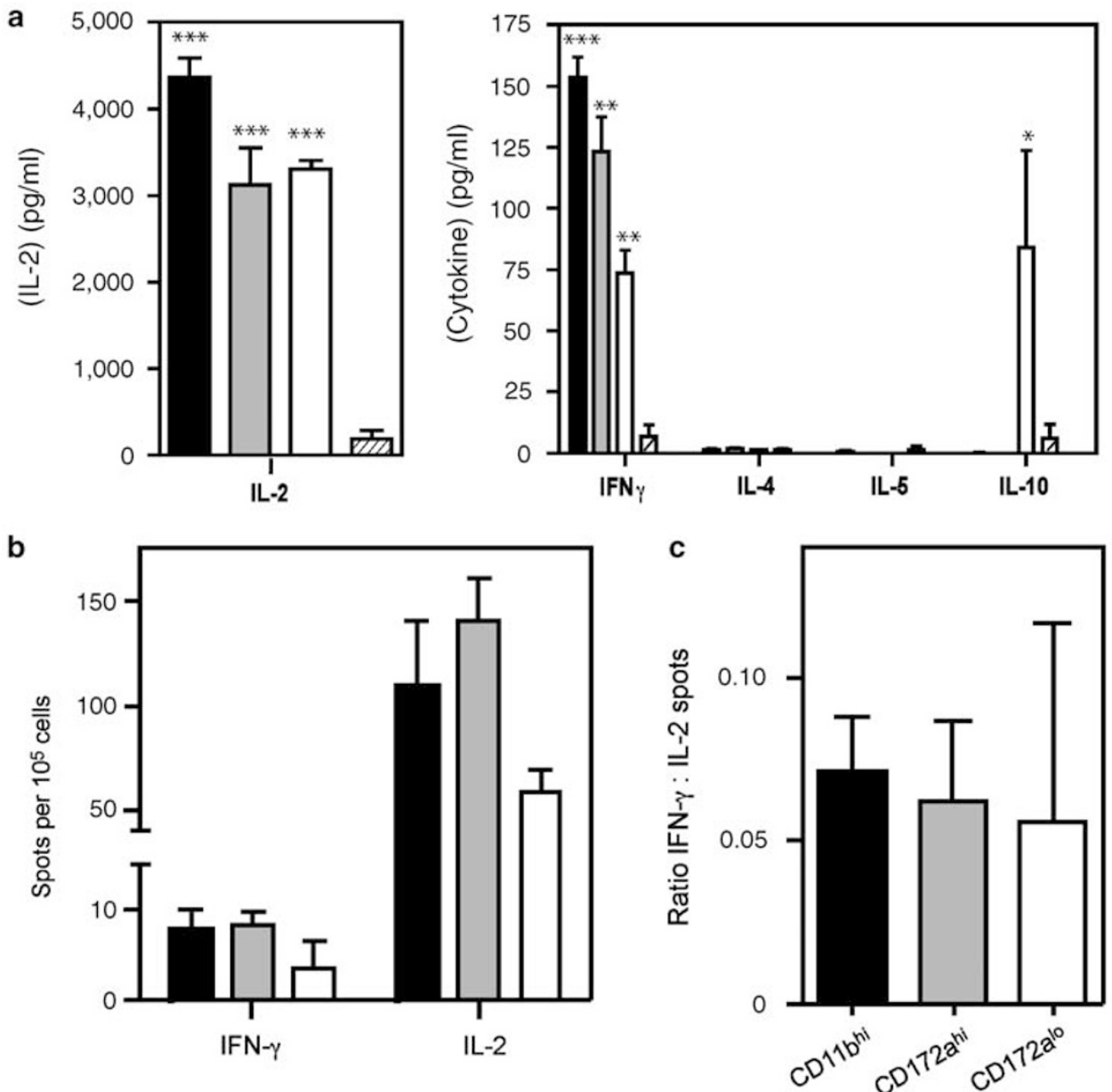

Figure 2 T-cells stimulated by steady-state L-DCs generate inflammatory, and Th1 cytokines. MLRs were set up using MoFlo-sorted CD4+ CD25CD45RC hi T lymphocytes and either CD11 b ${ }^{\text {hi }}$ (black-filled bars), CD172ahi (gray filled), or CD172alo (unfilled) sorted L-DC subsets, or L-DCs without added T cells (hatched bars). (a) After 3 days, supernatants were sampled from wells with similar DC numbers and their cytokine content measured using a rat cytokine multiplex kit. (b) MLRs were performed in ELISPOT plates, coated with antibodies specific for IL-2 or IFN- $\gamma$. After 5 days plates were washed, cytokine spots were developed and the number of cytokine-producing cells counted using an ELISPOT counter (Autoimmun Diagnostika, Strassberg, Germany). (c) The ratio of IFN- $\gamma$-/IL-2-producing cells was calculated from wells with matched cell populations, in triplicate. All data represent the mean and s.e.m. from three independent experiments. In (a) data were compared using one-way analysis of variance, and Tukey's multiple comparison test. Symbols represent significance of differences between samples and the "no T-cell" control. ${ }^{* \star *} P<0.001,{ }^{* *} P<0.01,{ }^{\star} P<0.05$. L-DC, lymph DC; MLR, mixed leucocyte reaction; ELISPOT, enzyme-linked immunospot assay.

DCs used for the initial stimulations. Secondly, irradiated RT1 ${ }^{\mathrm{u}}$ splenocytes from PVG-RT1 $1^{\mathrm{u}}$ rats were used. These "third party" splenocytes do not share their MHC haplotype with either the initial stimulators, or the responding $\left(\mathrm{RT}^{\mathrm{a}}\right) \mathrm{T}$ cells, and would be expected to stimulate a primary MLR in naive T cells. Finally, in separate experiments $\mathrm{T}$ cells were restimulated with paramethoxyamphetamine (PMA) and ionomycin. Proliferation of the $\mathrm{T}$ cells was measured by ${ }^{3} \mathrm{H}$-thymidine incorporation 3,4 , and 5 days after restimulation with splenocytes, and supernatants from all restimulated populations were assayed for cytokine content.

After restimulation with irradiated splenocytes, $\mathrm{RT} 1^{\mathrm{a}} \mathrm{T}$-cells proliferated more strongly in response to stimulation with "sensitizing" RT1 $1^{\mathrm{c}}$ splenocytes than to the RT1 $1^{\mathrm{u}}$ "third party" splenocytes (Figure 4a, b). This was true whichever subset of $\mathrm{RT} 1^{\mathrm{c}} \mathrm{L}$-DCs had been used to stimulate the primary MLR, and suggests that allo-specific T cells had expanded during the primary MLR. Furthermore, high levels of secreted inflammatory (IL-6) and Th1 (IFN- $\gamma$ ) cytokines in the supernatants of restimulated cultures again indicated that the surviving $\mathrm{T}$ cells were of an inflammatory Th1 phenotype (Figure 4c). Similar results were obtained when $\mathrm{RT} 1^{\mathrm{u}} \mathrm{T}$ cells were used as responders, initially cultured with $\mathrm{RT} 1^{\mathrm{c}} \mathrm{DCs}$ and then restimulated with RT1 $1^{\mathrm{c}}$ or RT1 $1^{\mathrm{a}}$ splenocytes (data not shown). To show that an important population of surviving $\mathrm{T}$ cells was not excluded from the splenocyte restimulation analysis, perhaps because of a lower affinity for the allo-MHC, the rested $\mathrm{T}$ cells were restimulated with PMA and ionomycin. Sixteen hours after PMA/ionomycin stimulation, supernatants were sampled and the concentration of secreted cytokines was measured. High concentrations of IL-2, IFN- $\gamma$, and IL- 9 were again generated, although after this type of stimulation the T-cells 
a
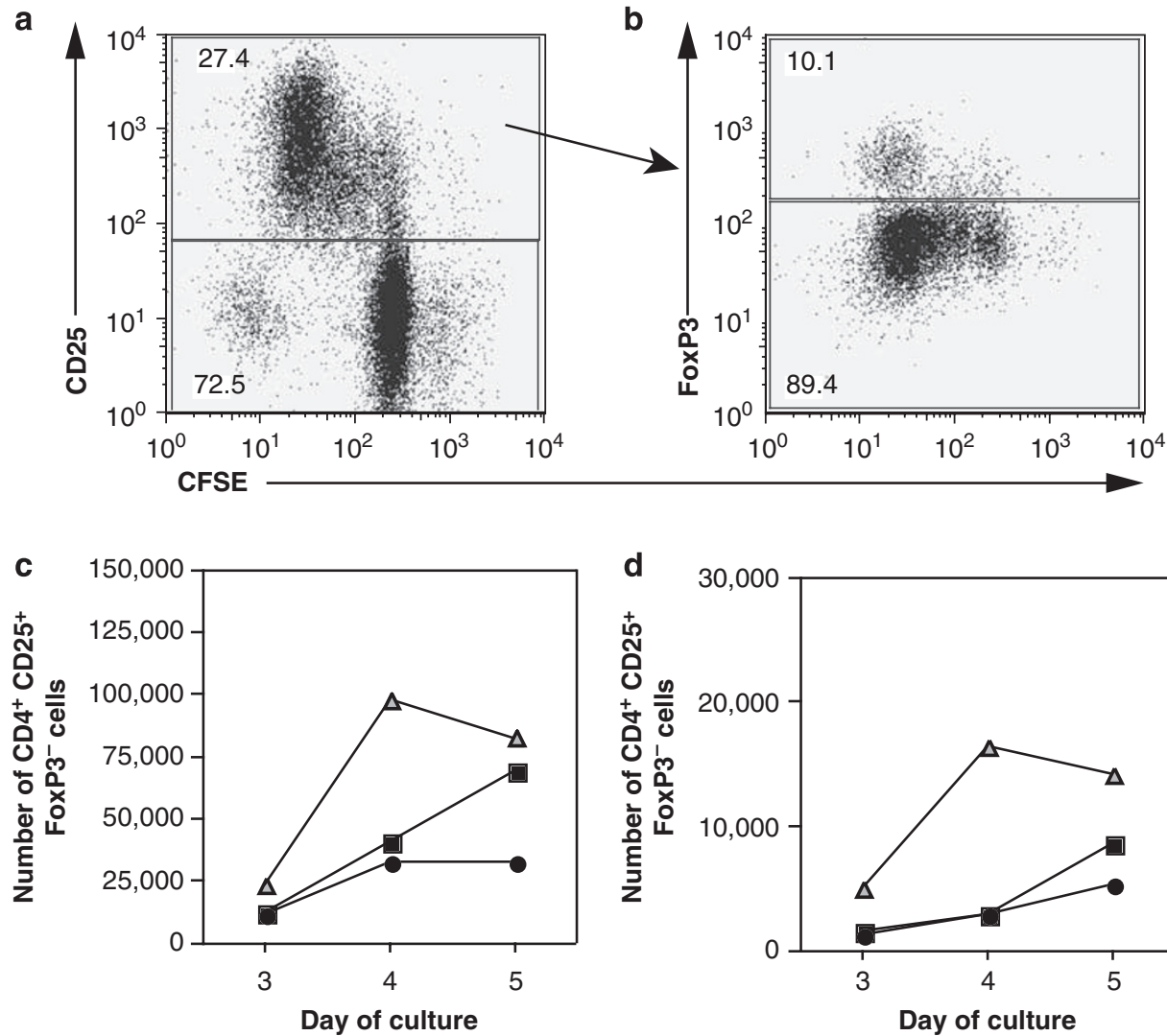

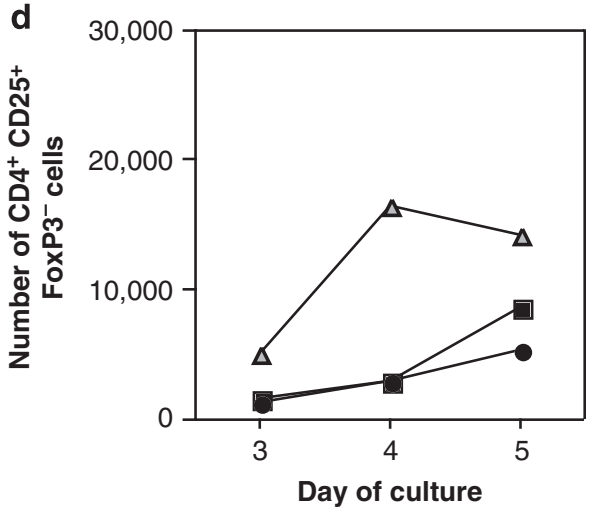

Figure 3 Steady-state L-DCs stimulate proliferation of both CD25+ FoxP3 ${ }^{-}$and CD25+ FoxP3 ${ }^{+}$allo T cells. Sorted $\left(2 \times 10^{6}\right) \mathrm{CD}^{+} \mathrm{CD}^{+} 5 \mathrm{RC} \mathrm{C}^{\text {hi }} \mathrm{CD} 25^{-}$ lymphocytes from DA rats were labeled with CFSE and mixed with sorted L-DC subsets $\left(2 \times 10^{4} \mathrm{~L}\right.$-DCs per well) and cultured. After 3 , 4 , or 5 days, cells were counted and stained for CD4, CD25, and FoxP3 before analysis by flow cytometry. After gating on CD4 $4^{+}$lymphocytes, cells were analyzed for expression of (a) CD25 and CFSE. The CD25 $5^{+}$cells were further analyzed according to their expression of (b) FoxP3 and CFSE. (a) and (b) show an example from day 5 after stimulation with CD172a hi L-DCs. From these data the numbers of cells with specific phenotypes that had undergone at least one division were determined (c) $\mathrm{CD} 4{ }^{+} \mathrm{CD} 25^{+}$FoxP3 ${ }^{-}$cells, (d) $\mathrm{CD} 4{ }^{+} \mathrm{CD}^{2} 5^{+}$FoxP3 ${ }^{+}$, after incubation with L-DCs of each subset: CD172ahi (blackfilled squares), CD11 bi (gray-filled triangles), CD172a ${ }^{\text {lo }}$ (circles). Representative plots are shown in (a) and (b). Data in (c) and (d) represent means from three independent experiments. L-DC, lymph DC; CFSE, carboxyfluorescein succinimidyl ester.

generated more IL-2 and IL-10 and less IL-6 than after allostimulation. In either case, there is no evidence that the majority of the restimulated $\mathrm{T}$ cells have adopted a noninflammatory Th2 phenotype.

\section{Cell-free lymph inhibits proliferation in MLR cultures}

To understand the effects that lymph itself might have on interactions between DCs and T cells, we added steady-state thoracic duct lymph from MLNX rats to MLR cultures at a range of concentrations from 1 to $5 \% \mathrm{v} / \mathrm{v}$. Although lymph does not contain detectable levels of transforming growth factor (TGF)- $\beta$ (Figure 5a), it inhibits proliferation of T cells at concentrations higher than $2 \%$ (Figure $5 \mathbf{b}$ ), without affecting cell viability. Similar results were obtained when proliferation was measured by analyzing dilution of CFSE in proliferating cells (data not shown). This inhibition was seen both when whole cell-free lymph was added to MLR cultures, and when the cell-free lymph had been centrifuged at high speed $(110,000 \mathrm{~g}, 2 \mathrm{~h})$ to enable removal of fat and fat-soluble compounds. Interestingly, addition of this fat-free lymph at low concentration (1\%) actually increased the level of proliferation in MLR cultures, indicating that lymph contains at least two factors with differential effects on T-cell responses.

Following these initial observations, we performed a number of experiments to further characterize the factor(s) in lymph responsible for inhibiting the MLR reaction. Passage of ultracentrifuged lymph through filters that only allow passage of molecules below defined molecular weights showed that the inhibitory factors have a mass of less than $3 \mathrm{kDa}$ (Figure 5c). We also discovered that the inhibitory factors are resistant to digestion with trypsin, and that they must be present at the time of the MLR reaction to exert their effect i.e., overnight incubation of DCs with lymph does not condition DCs or T cells to inhibit the MLR response (data not shown). In addition, we have found that lymph is able to inhibit MLR responses induced by lipopolysaccharide (LPS)matured DCs cultured from bone marrow, that the inhibitory factors are present in thoracic duct lymph whether animals have had their mesenteric lymph nodes removed, and that lymph from animals with indomethicin-induced intestinal inflammation is also strongly inhibitory in the MLR reaction (data not shown). 

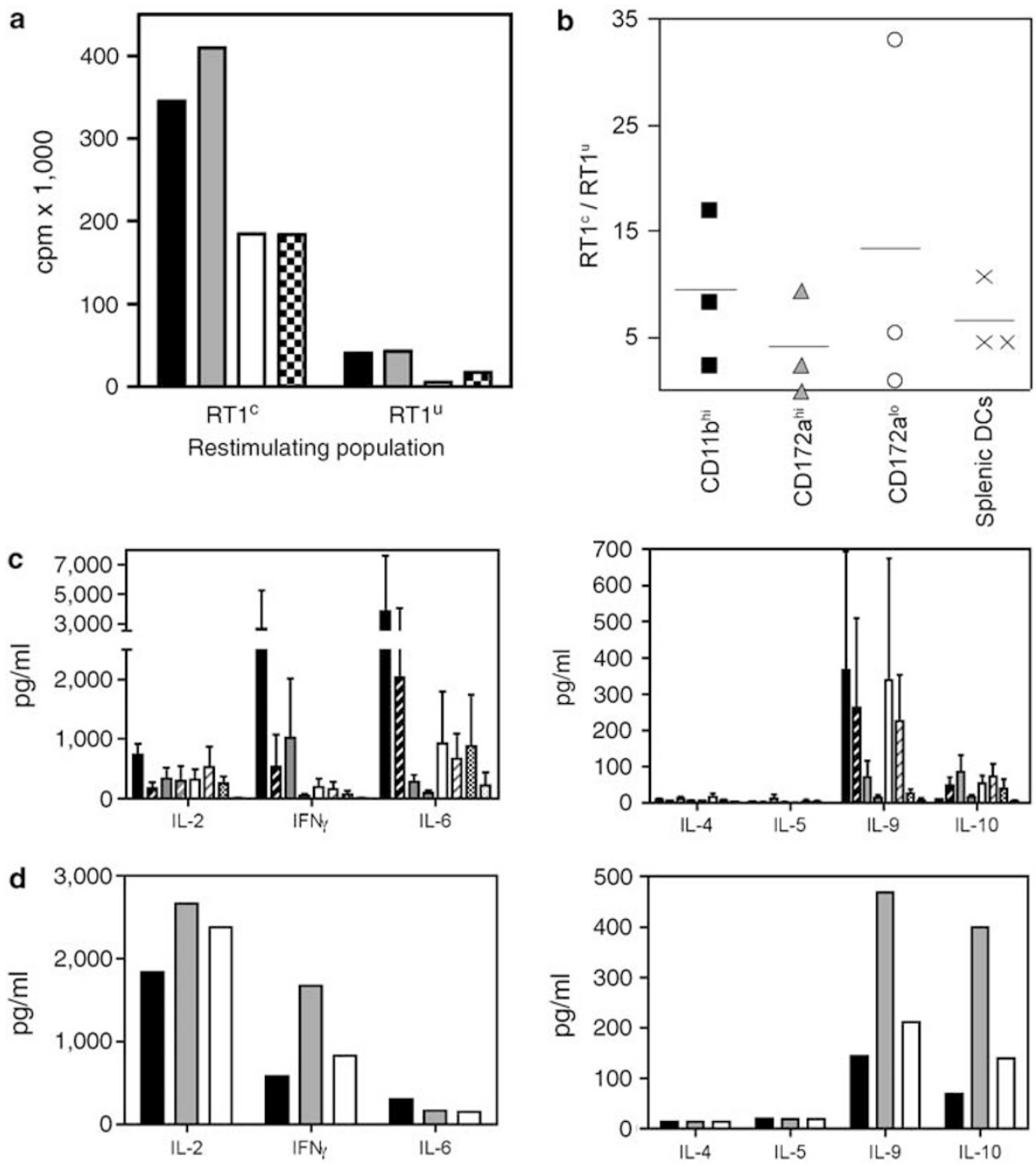

Figure 4 L-DC-stimulated allo-T cells retain their inflammatory Th1/Th2 phenotype after resting and restimulation. Sorted CD4 ${ }^{+}$CD45RC ${ }^{\text {hi }}$ CD25-

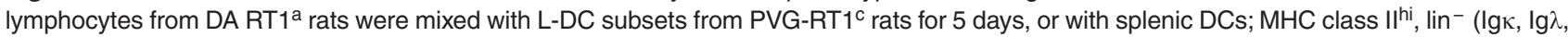
TCR, CD45R)) then washed, counted and rested in 20U/ml IL-2 for 3 days. They were then washed and counted again, before being mixed with PVG $\left(\mathrm{RT} 1^{\mathrm{c}}\right)$ or PVG-RT1 ${ }^{\mathrm{u}}$ splenocytes at a ratio of 1:1 for 3 days, or PMA and ionomycin for $16 \mathrm{~h}$. Proliferation of splenocyte-restimulated lymphocytes was measured by incorporation of tritiated thymidine for $16 \mathrm{~h}$ after day 3 . A representative plot of proliferation after splenocyte restimulation is shown in (a). Cells were initially stimulated with CD172a ${ }^{\text {hi }}$ (black filled), CD11 bhi (gray filled), or CD172alo (unfilled) L-DCs, or sorted splenic DCs (chequered). The summary of data from three independent experiments is shown in (b). Symbols represent individual measurements of the ratio between the proliferative responses to $\mathrm{RT} 1^{\mathrm{u}} \mathrm{vs}$. $\mathrm{RT} 1^{\mathrm{c}}$ restimulators after initial stimulation by each of the L-DC subsets: CD172a ${ }^{\text {hi }}$ (black-filled squares), CD11b ${ }^{\text {hi }}$ (gray-filled triangles), CD172alo (open circles), control splenic DCs $(x)$. Three days after MLR cultures were set up (c), or 16h after PMA/ionomycin stimulation (d), supernatants were sampled and analyzed by multiplex cytokine array; cells initially stimulated by the different DC subsets are represented as follows: CD11 bi L-DCs (black filled), CD172a ${ }^{\text {hi }}$ L-DCs (gray filled), CD172alo L-DCs (unfilled), splenic DCs (chequered). RT1

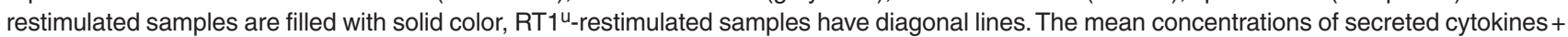
s.e.m. from three independent experiments are shown in (c) and one representative experiment from three is shown in (d). L-DC, Lymph DC; DC, dendritic cell; PMA, paramethoxyamphetamine.

\section{DISCUSSION}

DCs are not only uniquely able to activate naive $\mathrm{CD}^{+} \mathrm{T}$ lymphocytes physiologically, they also influence the developmental fate of the expanding clones. ${ }^{12,13}$ In the steady state around 800,000 DCs migrate each day from the rat small intestine to the mesenteric lymph nodes. ${ }^{14}$ These migrating cells carry selfantigens, ${ }^{15}$ they are all CCR7-positive, ${ }^{14}$ and migrate from the intestine; ${ }^{11}$ from the lamina propria, and perhaps also from the Peyer's patches. These DCs are required for the maintenance of oral tolerance. ${ }^{8}$ A mechanism by which migrating DCs may mediate tolerance to intestinal antigens has been described, where DCs cultured from human blood monocytes and conditioned with TSLP are unable to stimulate the production of inflammatory cytokines or IFN- $\gamma$ from allo-specific T cells. ${ }^{5}$

To understand whether the DCs that actually migrate from the intestine have the same properties as those expanded from precursors in vitro, we first tested the ability of MoFlo-sorted subsets of DCs from intestinal lymph to induce proliferation from 

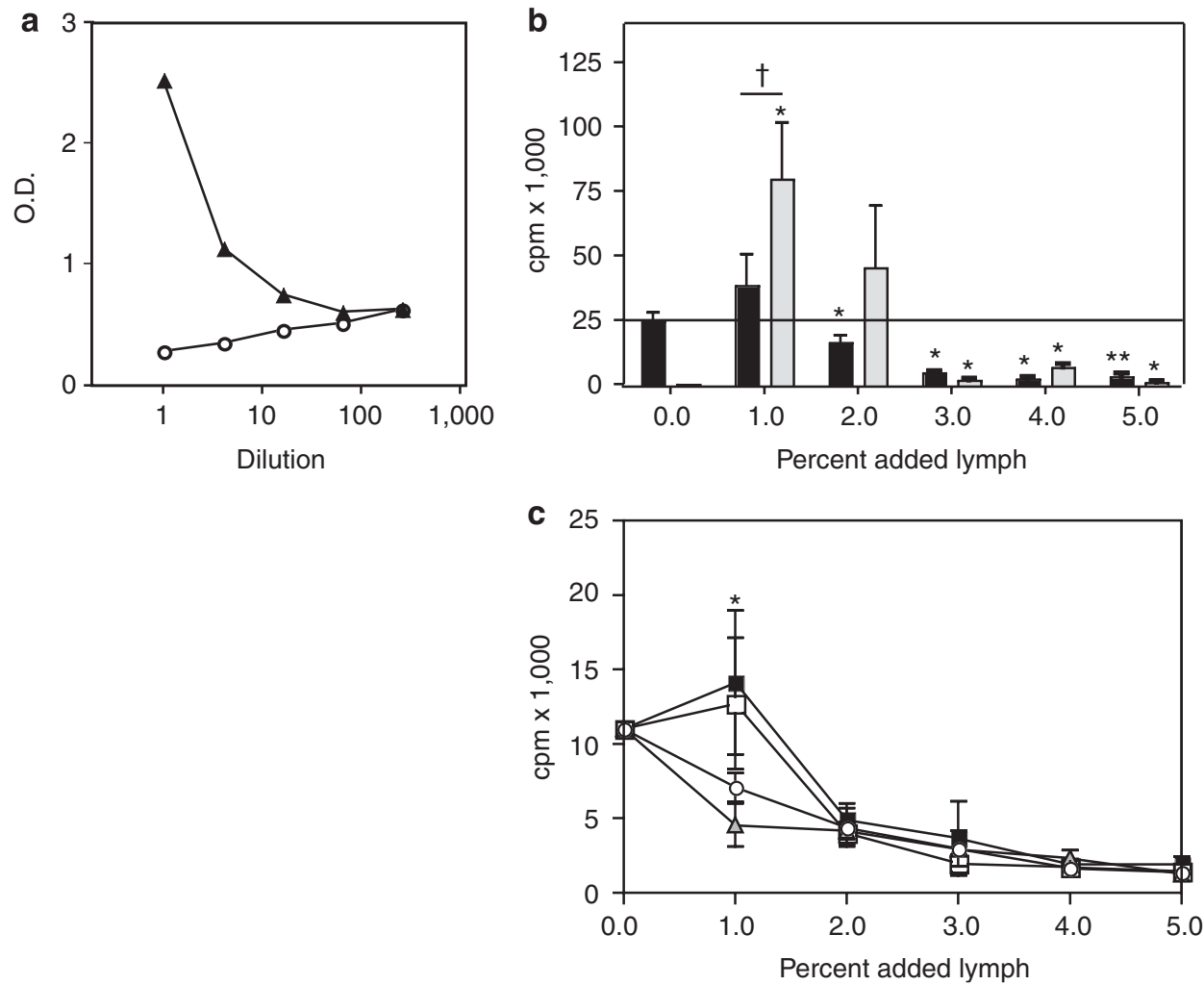

Figure 5 Cell-free lymph inhibits proliferation in MLRs, but does not contain detectable TGF- $\beta$. (a) The TGF- $\beta$ concentration in thoracic duct lymph was analyzed using a TGF- $\beta$-specific ELISA. Recombinant TGF- $\beta$ generated a strong signal (closed triangles), whereas lymph samples were negative at all dilutions (open circles). (b) Thoracic duct lymph from MLNX rats was added at a range of concentrations to MLR cultures of $1 \times 10^{5}$ splenocytes each, from $\mathrm{RT}^{\mathrm{c}}{ }^{\mathrm{C}}$ and $\mathrm{RT} 1^{\mathrm{u}}$ animals. The lymph was either prepared by low-speed centrifugation $(500 \mathrm{~g}, 5 \mathrm{~min})$ to remove cells (filled bars), or with an additional ultracentrifugation step $(100,000 \mathrm{~g}, 2 \mathrm{~h}$ ) to enable removal of fat-soluble compounds (gray bars). Proliferation of splenocytes was measured by incorporation of tritiated thymidine for $16 \mathrm{~h}$ after day 5 . In (b) means of triplicates are represented \pm s.e.m., from one representative experiment of three independent replicates. The horizontal line represents the mean proliferation in the absence of added lymph. Samples were compared using a two-tailed $t$-test. Significant differences between whole and low fat lymph are indicated by ${ }^{\dagger} P<0.05$. Significant differences from the no lymph control are indicated by ${ }^{*} P<0.05$. (c) Ultracentrifuged lymph $(100,000 \mathrm{~g}, 1 \mathrm{~h})$ was separated into fractions by filtration through filters with defined molecular weight cutoffs, and tested for its capacity to affect proliferation in MLR reactions. MLRs were treated with titrated doses of ultracentrifuged lymph (filled squares), ultracentrifuged lymph $<30 \mathrm{kDa}$ (open squares), ultracentrifuged lymph $<10 \mathrm{kDa}$ (gray triangles), or ultracentrifuged lymph $<3 \mathrm{kDa}$ (open circles). In (c), means of triplicates are represented \pm s.e.m., from one of two similar experiments. Samples were analyzed by two-way analysis of variance, and the only significant difference $(P<0.05)$ was seen between ultracentrifuged lymph and ultracentrifuged lymph $<3 \mathrm{kDa}$, when added at $1 \%$. This is indicated by $a^{*}$. MLR, mixed leucocyte reaction; TGF, transforming growth factor; ELISA, enzyme-linked immunosorbent assay.

$\mathrm{CD}^{+} \mathrm{T}$ cells. We chose to study this DC population because DCs from intestinal lymph are obtained with the fewest possible in vitro manipulation; they flow from the animal suspended in lymph, and are collected on ice. All the subsequent purification steps are carried out at $0-4{ }^{\circ} \mathrm{C}$ without any enzymatic digestion. Although we cannot prove that these DCs have the same phenotype as the cells in their physiological environment, we have previously shown that they express the same surface phenotype both before and after enrichment and purification. ${ }^{16}$ Here, we show that both of the CD172a ${ }^{\text {hi }}$ L-DC subsets stimulated vigorous proliferative responses (Figure 1c). These proliferation results are similar to those previously observed using L-DCs, ${ }^{9}$ although in these earlier experiments only two L-DC subsets had been identified. It is possible that the CD172a ${ }^{\text {lo }} \mathrm{L}$-DCs stimulate less proliferation because they survive less well in culture. ${ }^{9}$ Our results were enhanced by experiments that showed that the level of proliferation increased when $\mathrm{CD} 4{ }^{+} \mathrm{CD} 25^{+}$cells were removed from the pool of responding $\mathrm{T}$ cells (Figure 1d). As purified CD25 ${ }^{+} \mathrm{T}$ cells did not proliferate in response to alloDCs (data not shown), this suggests that the $\mathrm{CD} 25^{+} \mathrm{CD} 4^{+}$cells in these experiments reduced the level of proliferation induced by the DCs. We also showed that sorted naive $\left(\mathrm{CD} 45 \mathrm{RC}^{\mathrm{lo}} \mathrm{CD} 25^{-}\right.$ $\mathrm{CD}^{+}$) T-cells proliferated more strongly than their CD45RC ${ }^{\text {hi }}$ counterparts (Figure 1e, f), as expected. ${ }^{17}$ These data clearly show that L-DCs are capable of stimulating strong proliferative from lymph node $\mathrm{T}$ cells, and led us to examine the cytokines produced by the T-cell cultures.

When naive $\mathrm{CD} 4{ }^{+} \mathrm{T}$ lymphocytes were mixed with purified steady-state L-DC subsets for 3, 4, or 5 days, both CD172ahi DC subsets induced high concentrations of both IL- 2 and IFN- $\gamma$, but no IL- 4 or IL-5, indicating that the DCs induced an inflammatory Th1 phenotype in the responding T cells (Figure 2a). On occasion, the CD172 $\mathrm{a}^{\text {lo }}$ DC subset also induced expression of IL-10 (Figure 2a), indicating that some of the T-cells activated 
by this DC population might have some regulatory functions. IL-10 was not produced in all experiments, however, making these data difficult to interpret. IL-10 was, however, consistently generated when naive $\mathrm{T}$ cells were stimulated by allogeneic splenocytes in the presence of additional MoFlo-sorted Ig $\kappa^{+} / \operatorname{Ig} \lambda^{+}$B lymphocytes.

An ELISPOT was used to determine the proportion of naive T-cells stimulated to produce cytokines by each of the DC populations. The input $\mathrm{T}$ cells $(0.5-1.5 \%)$ were induced to secrete detectable amounts of IL-2 (Figure 2b), a proportion similar to that seen in human MLR experiments. ${ }^{18}$ Although the CD172a $\mathrm{a}^{\text {hi }}$ L-DC cultures generated higher numbers of IL-2-secreting cells, the ratio of IL-2-/IFN- $\gamma$-producing cells was the same (20:1) after stimulation by each of the L-DC populations (Figure 2c), suggesting that the differences between the subsets might be quantitative rather than qualitative. Our interpretation is that a subset of IL-2-producing cells also secretes IFN- $\gamma$. The reduced induction of proliferation, IL- 2 and IFN- $\gamma$ production by the CD172a ${ }^{\text {lo }} \mathrm{L}-\mathrm{DC}$ population may reflect the fact that this population survives less well in culture, ${ }^{9}$ reducing the number of DC available to stimulate DCs after the initial period. These results show that L-DCs stimulate strong proliferation from naive $\mathrm{T}$ cells and generate cytokines indicative of an inflammatory response characterized by Th1 cytokines, rather than stimulating noninflammatory $\mathrm{Th} 2 \mathrm{~T}$-cell responses.

To further investigate the characteristics of the induced T-cell populations, the expression of CD25 and FoxP3 were examined in CFSE-labeled naive T cells, stimulated with sorted allogeneic L-DC subsets. All three DC subsets stimulated an increase in the number of "activated" CD4 ${ }^{+} \mathrm{CD} 25^{+} \mathrm{FoxP}^{-} \mathrm{T}$ cells (Figure $3 c)$ and "regulatory" $\mathrm{CD} 4{ }^{+} \mathrm{CD} 25^{+} \mathrm{FoxP} 3^{+}$cells in the cultures (Figure 3d). The CD11b $b^{\text {hi }}$ L-DCs induced the highest numbers of both T-cell populations. The percentage of CD25 ${ }^{+} \mathrm{CFSE}^{\text {lo }}$ activated cells (3.5-4.2\%) is somewhat higher than the percentage of IL-2-producing cells detected in the ELISPOT assay in Figure 2. This difference is likely to be caused by technical differences between the two assays. For instance, recently divided cells, cells in close proximity to one another, and cells secreting only low levels of IL-2, are all unlikely to be detected by the ELISPOT assay. It is not clear whether the regulatory cells were generated de novo from FoxP3- precursors, or whether they arise from the expansion of a small population of preexisting FoxP3 ${ }^{+}$cells. Such expansion of regulatory T cells in response to stimulation by DCs, ${ }^{19}$ particularly CD $103^{+}$DCs has been reported previously. ${ }^{20,21}$ In our hands FoxP3 ${ }^{+} \mathrm{T}$ cells are generated in the absence of exogenously added IL-2 or exogenously added TGF- $\beta$. They do not appear to have a strong suppressive effect in the cultures, as the $\mathrm{CD} 25^{+}$FoxP3 ${ }^{-}$T cells are strongly proliferative, in an environment rich in IFN- $\gamma$.

Our data from the primary MLRs demonstrate that steadystate migrating L-DCs do not induce a "Th2-tolerogenic" phenotype in responding naive allogeneic $\mathrm{T}$ cells. It was, however, possible that polarization might only become apparent upon restimulation of these cells, or that regulatory $\mathrm{T}$ cells might predominate following restimulation. We showed that not only did the allo-specific T-cells expand during the primary MLR, but also that the cells which were restimulated, again secreted high levels of IL-2, IL-6, and IFN- $\gamma$ (Figure 4c). These data again demonstrate that $\mathrm{T}$ cells which interact with migrating intestinal DCs do not adopt a noninflammatory Th2 phenotype.

Contrary to the predictions resulting from experiments using human monocyte-derived DCs, or CD11 $\mathrm{c}^{+}$cells from samples of human intestine, ${ }^{5}$ we show that steady-state L-DCs migrating from the intestine in lymph are not conditioned to induce a noninflammatory Th2 phenotype in the T cells they stimulate, but instead stimulate strong proliferation and an inflammatory Th1 response. These inflammatory Th1 characteristics are retained after the T cells have been rested and restimulated. As expected, T-cells induced to divide by the L-DCs express CD25; a minority also express the FoxP3 transcription factor, a hallmark of regulatory T-cell populations.

Our data were generated using the most appropriate population of cells, the steady-state migrating intestinal DCs responsible for the maintenance of oral tolerance. Currently, sufficient numbers of these L-DCs can only be obtained from rats. The results presented here are consistent with previous experiments where tolerogenic DCs extracted from murine intestinal lamina propria were able to induce antigen-specific responses from naive $\mathrm{CD} 4^{+} \mathrm{T}$ cells. ${ }^{22} \mathrm{We}$ are not able to repeat these experiments using cells purified from rat intestinal tissue because suitable markers for rat lamina propria DCs have not been identified. CD11c is not expressed at a high enough level for it to be used to enrich DCs, and both MHC class II and CD103 are expressed by intraepithelial lymphocytes in this tissue (S.M., U.Y., unpublished observations).

The cytokine expression and restimulation data presented here represent novel and important advances in understanding steady-state L-DC function. ${ }^{9,11}$ We confirm that the CD172a $\mathrm{a}^{\text {lo }}$ subset stimulates less proliferation in MLRs, and additionally show that this subset generates fewer IL-2- and IFN- $\gamma$-producing cells, although the ratio of IL-2-/IFN- $\gamma$-producing cells is similar for all stimulators. Culture of $\mathrm{T}$ cells with different $\mathrm{DC}$ subsets does not polarize them to generate dramatically different cytokine profiles when restimulated, although $\mathrm{T}$ cells initially exposed to CD172 $\mathrm{a}^{\mathrm{lo}}$ DCs produce less IFN- $\gamma$ and more IL-10 than those stimulated by the other subsets, and cells initially stimulated by CD172 $\mathrm{a}^{\text {lo }}$ DCs generate less IFN- $\gamma$ on restimulation with allo-splenocytes. Therefore, the CD172a $\mathrm{a}^{\text {lo }} \mathrm{L}-\mathrm{DC}$ subset is less able to stimulate proliferation and Th1 cytokine production form naive $\mathrm{T}$ cells. Although these data are consistent with its previously described role as a "tolerogenic" subset of L-DCs, ${ }^{10}$ they are, however, not better than the other L-DC subsets at stimulating proliferation of $\mathrm{CD} 25^{+} \mathrm{FoxP}^{+} \mathrm{T}$ cells. They might therefore be better described as poor stimulators, rather than as tolerogenic DCs.

These data present a clear and consistent picture of the behavior of the DCs that migrate in steady-state intestinal lymph. They also raise an important question: if steady-state L-DCs stimulate naive $\mathrm{T}$ cells to proliferate and secrete inflammatory cytokines, how are they also able to maintain tolerance to intestinal antigens in vivo ${ }^{8}$ We hypothesize that although migrating L-DCs activate naive $\mathrm{T}$ cells in vitro, they do not activate $\mathrm{T}$ cells in vivo. We 
propose two potential mechanisms. Firstly, in the steady state the CD172a ${ }^{\text {hi }}$ L-DC subsets, which stimulate stronger proliferation of naive $\mathrm{T}$ cells, might not reach the ideal location within the mesenteric node. Although the CD172a ${ }^{\text {lo }}$ L-DC subset ${ }^{10}$ can be found throughout the T-cell area of the mesenteric lymph nodes, CD172a ${ }^{\text {hi }}$ DC subsets only migrate into the center of the T-cell area after stimulation by molecules such as lipopolysaccharide ${ }^{4}$ or Etx. ${ }^{14}$ Secondly, lymph-borne compounds are likely to bathe lymph nodes, continually providing signals to condition the cells interacting therein. In the steady state intestinally derived factors delivered by intestinal lymph may affect activation of naive $\mathrm{T}$ cells in the mesenteric lymph nodes. Indeed, we have observed that addition of steady-state lymph to MLR cultures inhibits proliferation of $\mathrm{T}$ cells (Figure $\mathbf{5 b}$ ), without affecting cell viability. We speculated, therefore, that intestinal lymph might contain intestinally generated immunomodulatory cytokines such as TSLP $^{5}$ or TGF- $\beta{ }^{23}$ Unfortunately, we cannot detect TGF- $\beta$ in rat lymph (Figure $5 \mathbf{b}$ ), and know of no reagents for the detection of rat TSLP. Additional experiments showed that the inhibitory factors have a molecular weight of less than $3 \mathrm{kDa}$ (Figure 5c), that it is not toxic to DCs or T cells, and it cannot be used to pretreat DCs or T cells; it must be present in the MLR culture to exert its inhibitory effect (data not shown).

Current techniques for collection of DCs from pseudo-afferent intestinal lymph require dilution of the fluid by continuous intravenous infusion of the donor animal, hampering detection of soluble factors. Future investigations will therefore involve development of improved lymph collection techniques to enable identification of immunomodulatory molecules.

\section{METHODS}

\section{Animals}

All rats were males of PVG $\left(\mathrm{RT}^{\mathrm{c}}\right), \mathrm{PVG}-\mathrm{RT} 1^{\mathrm{u}}\left(\mathrm{RT} 1^{\mathrm{u}}\right)$, or DA $\left(\mathrm{RT} 1^{\mathrm{a}}\right)$ strains, bred at the Sir William Dunn School of Pathology under specific pathogen free conditions. Experiments were carried out under the authority of licenses issued by the Home Office, UK.

\section{Cell preparation and purification}

L-DCs were collected from the thoracic ducts of mesenteric lymphadenectomized (MLNX) PVG-RT1 ${ }^{\mathrm{c}}$ rats as described previously. ${ }^{24}$ Cells were washed in complete medium: RPMI with $5 \%$ fetal bovine serum, 2-mercaptoethanol, penicillin, streptomycin, and L-glutamine (Invitrogen, Paisley, UK). All steps were performed on ice. Where stated, L-DCs were enriched using anti-CD103 microbeads (Miltenyi Biotec, Bisley, UK). For fluorescence-activated cell sorting, cells were labeled with antibodies against CD103 (OX62; M. Puklavec, Cellular Immunology Unit, Sir William Dunn School of Pathology, Oxford, UK), MHC class II (OX6-PerCP; BD Biosciences, San Jose, CA), CD11b-PE (OX42-PE; Serotec, Kidlington, UK), and CD172a (SIRP $\alpha$; OX41; M. Puklavec). OX41 and OX62 antibodies were conjugated using the Alexa Fluor 647 and Alexa Fluor 488 protein labeling kits, respectively (Molecular Probes, Eugene, OR). L-DCs, expressing high levels of MHC class II and CD103, were sorted into three subsets based on their expression of $\mathrm{CD} 11 \mathrm{~b}$ and CD172a ${ }^{11}$ using a MoFlo sorter (DakoCytomation, Carpinteria, CA).

\section{Mixed lymphocyte reactions}

T cells from cervical and mesenteric lymph nodes, were sieved through a $100 \mu \mathrm{m}$ cell strainer to generate a single cell suspension. Cells were labeled with antibodies specific for CD4 (OX35-PE-Cy5), CD25 (OX39-PE; BD
Biosciences), and CD45RC (OX22, M. Puklavec). OX22 was conjugated to Alexa-647 using a protein labeling kit (Molecular Probes). CD ${ }^{+}$, $\mathrm{CD}^{2} 5^{-}, \mathrm{CD} 45 \mathrm{RC}$ hi lymphocytes were purified using a MoFlo sorter (DakoCytomation). In some experiments, T cells were labeled with $1 \mu \mathrm{M}$ CFSE (Molecular Probes), before being mixed with APCs. MoFlo-sorted LN cells were incubated in 96-well or 24-well plates with MoFlo-sorted L-DC subsets, as described in figure legends.

In some experiments, after 5 days $1 \mu \mathrm{Ci}^{3} \mathrm{H}$-thymidine was added to each well, and cultures left for a further $16 \mathrm{~h}$. Cells were harvested and thymidine incorporation measured using a $\beta$-plate counter (PerkinElmer, Boston, MA). In other experiments, cells were harvested after 3, 4, or 5 days, counted, and stained for flow cytometry. These cells were also fixed and stained intracellularly with an antibody specific for FoxP3 or IgG2a$\kappa$-APC isotype control (eBioscience, San Diego, CA). A Cyan cytometer (Dako, Ely, UK) was used to generate data, which were analyzed using FlowJo software (TreeStar, Ashland, OR).

For restimulation assays a DC/T-cell ratio of 1:100 was used, as this ratio generated optimum proliferation in pilot experiments. After 5 days cells were harvested, washed, counted and rested for 3 days at a density of $1 \times 10^{6}$ cells per $\mathrm{ml}$ in complete medium supplemented with $20 \mathrm{U} / \mathrm{ml}$ IL-2 (M. Puklavec). They were then restimulated either for 3 days using irradiated splenocytes from (i) PVG-RT1 $1^{\mathrm{c}}$ or (ii) PVG-RT1 $1^{\mathrm{u}}$ rats at a splenocyte/T-cell ratio of 1:1, or iii) by incubation for $16 \mathrm{~h}$ with $5 \mathrm{ng} / \mathrm{ml}$ PMA and $500 \mathrm{ng} / \mathrm{ml}$ ionomycin (Sigma, Poole, UK). After restimulation, supernatants were collected and in some experiments, proliferation was measured by analyzing incorporation of ${ }^{3} \mathrm{H}$-thymidine, as above.

The MLR cultures used to demonstrate the antiproliferative effects of lymph contained red blood cell-depleted suspensions of cells from mesenteric and cervical lymph nodes. Cells $\left(1 \times 10^{5}\right)$ each from PVG and DA rats were used. Proliferation was assessed by thymidine incorporation after 5 days.

\section{Cytokine analysis}

The concentrations of secreted cytokines were measured using a rat cytokine multiplex array (Millipore, Billarica, MA). Samples were analyzed using a Bio-Plex multiplex analysis system (Bio-Rad, Hemel Hempstead, UK). ELISPOTs for IL-2 and IFN- $\gamma$ were performed using rat ELSPOT kits (R\&D Systems, Abingdon, UK).

\section{ACKNOWLEDGMENTS}

We are especially thankful to Nigel Rust for expert assistance with cell sorting, and to Allan Mowat for critical reading of the article. This study has been supported by a grant from the BBSRC to G. MacPherson and S. Milling.

\section{DISCLOSURE}

The authors declared no conflict of interest.

(C) 2009 Society for Mucosal Immunology

\section{REFERENCES}

1. Mowat, A.M. Anatomical basis of tolerance and immunity to intestinal antigens. Nat. Rev. Immunol. 3, 331-341 (2003).

2. Pugh, C.W., MacPherson, G.G. \& Steer, H.W. Characterization of nonlymphoid cells derived from rat peripheral lymph. J. Exp. Med. 157, 1758-1779 (1983).

3. Wilson, N.S. et al. Normal proportion and expression of maturation markers in migratory dendritic cells in the absence of germs or Toll-like receptor signaling. Immunol. Cell Biol. 86, 200-205 (2008).

4. Turnbull, E., Yrlid, U., Jenkins, C.D. \& MacPherson, G. Intestinal dendritic cell subsets: differential effects of systemic TLR4 stimulation on migratory fate and activation in vivo. J. Immunol. 174, 1374-1384 (2005).

5. Rimoldi, M. et al. Intestinal immune homeostasis is regulated by the crosstalk between epithelial cells and dendritic cells. Nat. Immunol. 6, 507-514 (2005). 
6. Iliev, I.D., Matteoli, G. \& Rescigno, M. The yin and yang of intestinal epithelial cells in controlling dendritic cell function. J. Exp. Med. 204, 2253-2257 (2007).

7. Liu, L.M. \& MacPherson, G.G. Antigen acquisition by dendritic cells: intestinal dendritic cells acquire antigen administered orally and can prime naive T cells in vivo. J. Exp. Med. 177, 1299-1307 (1993).

8. Worbs, T. et al. Oral tolerance originates in the intestinal immune system and relies on antigen carriage by dendritic cells. J. Exp. Med. 203, 519-527 (2006).

9. Liu, L., Zhang, M., Jenkins, C. \& MacPherson, G.G. Dendritic cell heterogeneity in vivo: two functionally different dendritic cell populations in rat intestinal lymph can be distinguished by CD4 expression. J. Immunol. 161, 1146-1155 (1998)

10. Huang, F.P. et al. A discrete subpopulation of dendritic cells transports apoptotic intestinal epithelial cells to T cell areas of mesenteric lymph nodes. J. Exp. Med. 191, 435-444 (2000).

11. Yrlid, U. et al. A distinct subset of intestinal dendritic cells responds selectively to oral TLR7/8 stimulation. Eur. J. Immunol. 36, 2639-2648 (2006).

12. Palucka, K. \& Banchereau, J. How dendritic cells and microbes interact to elicit or subvert protective immune responses. Curr. Opin. Immunol. 14, 420-431 (2002).

13. Manickasingham, S.P., Edwards, A.D., Schulz, O. \& Reis e Sousa, C. The ability of murine dendritic cell subsets to direct Thelper cell differentiation is dependent on microbial signals. Jan 33, 101-107 (2003).

14. Milling, S.W., Yrlid, U., Jenkins, C., Richards, C.M., Williams, N.A., MacPherson, G. Regulation of intestinal immunity: effects of the oral adjuvant Escherichia coli heat-labile enterotoxin on migrating dendritic cells. Eur. J. Immunol. 37, 87-99 (2007).

15. Scheinecker, C., McHugh, R., Shevach, E.M. \& Germain, R.N. Constitutive presentation of a natural tissue autoantigen exclusively by dendritic cells in the draining lymph node. J. Exp. Med. 196, 1079-1090 (2002).

16. Yrlid, U., Milling, S.W. Miller, J.L., Cartland, S., Jenkins, C.D. \& MacPherson, G.G. Regulation of intestinal dendritic cell migration and activation by plasmacytoid dendritic cells, TNF-alpha and type 1 IFNs after feeding a TLR7/8 ligand. J. Immunol. 176, 5205-5212 (2006).

17. Chen, B.J., et al. Inability of memory T cells to induce graft-versus-host disease is a result of an abortive alloresponse. Blood 109, 3115-3123 (2007).

18. Hernandez-Fuentes, M.P., Stevenson, S., Barroso-Herrera, O. \& Lechler, R.I. Establishing the optimal method to estimate the frequencies of alloreactive CD4+ T cells. Transplant. Proc. 34, 2855-2857 (2002).

19. Yamazaki, S. et al. Effective expansion of alloantigen-specific Foxp3+ CD25+CD4+ regulatory $T$ cells by dendritic cells during the mixed leukocyte reaction. Proc. Natl. Acad. Sci. USA 103, 2758-2763 (2006).

20. Coombes, J.L. et al. A functionally specialized population of mucosal CD103+ DCs induces Foxp3+ regulatory T cells via a TGF-\{beta $\}$ and retinoic acid dependent mechanism. J. Exp. Med. 204, 1757-1764 (2007).

21. Sun, C.M. et al. Small intestine lamina propria dendritic cells promote de novo generation of Foxp3T reg cells via retinoic acid. J. Exp. Med. 204, 1775-1785 (2007).

22. Chirdo, F.G., Millington, O.R., Beacock-Sharp, H. \& Mowat, A.M. Immunomodulatory dendritic cells in intestinal lamina propria. Eur. J. Immunol. 35, 1831-1840 (2005).

23. Barnard, J.A., Beauchamp, R.D., Coffey, R.J. \& Moses, H.L. Regulation of intestinal epithelial cell growth by transforming growth factor type beta. Proc. Natl. Acad. Sci. USA 86, 1578-1582 (1989).

24. MacPherson, G.G. Properties of lymph-borne (veiled) dendritic cells in culture. I. Modulation of phenotype, survival and function: partial dependence on GM-CSF. Immunology 68, 102-107 (1989). 